\title{
Transfer of occasion setting: The role of generalization decrement
}

\author{
CHARLOTTE BONARDI \\ University of York, York, England
}

\begin{abstract}
In Experiment 1, pigeons were trained on two feature-positive discriminations. A transfer test examined whether the feature from one discrimination enhanced responding to the target from the other. Transfer was obtained, but it was incomplete; the feature produced less responding to the transfer target than to its own. Experiments 2 and 3 examined whether this attenuation of responding was the product of generalization decrement induced by the novel combination of feature and target on transfer trials. Birds were trained on a pair of pseudo-occasion-setting discriminations in which each target was reinforced whether or not it was preceded by its feature. In a subsequent test, there was no loss of responding when novel combinations of features and targets were introduced; on the contrary, responding was, if anything, enhanced in this condition. This suggests that imperfect transfer is not due to generalization decrement but to the fact that an occasion setter is specific to its target stimulus.
\end{abstract}

An occasion setter is a cue signaling that some other stimulus will be followed by reinforcement; animals can learn this relationship, responding more to the stimulus when it is preceded by the occasion setter than when it is presented alone. This modulation of the association between the conditioned stimulus (CS) and unconditioned stimulus (US) is independent of any direct association between the occasion setter and reinforcement (e.g., Holland, 1983; Rescorla, 1985; Ross \& Holland, 1981, 1982). One interpretation of this phenomenon is that the occasion setter acts on the entire CS-US association, increasing the ease with which the CS can activate the representation of the US, and hence elicit a conditioned response (e.g., Bonardi, 1989; Bouton, 1990; Holland, 1983). As this account supposes that the occasion setter acts on a particular CS-US association, it follows that the effect of the occasion setter will be specific to the CS with which it was trained-it should not show transfer and affect responding to a second CS.

The data from experiments undertaken to test this prediction allow no clear conclusion, however. Evaluating transfer involves comparing the effect of the occasion setter on its own CS with its effect on a second CS that, although as similar as possible to the original CS in terms of its training history, has not been trained with that particular occasion setter. In a number of experiments, this has been done by examining the effect of the occasion setter on responding to a transfer CS that has been trained in an occasion-setting discrimination with a different oc-

This research was financed by a grant from the Science and Engineering Research Council. I would like to thank Geoff Hall for much helpful discussion and several anonymous reviewers for their useful comments. Correspondence regarding the paper should be addressed to C. Bonardi, Department of Psychology, University of York, Heslington, York YO1 5DD, England (e-mail: cb2@york.unix). casion setter. The typical finding of such studies is that the occasion setter will enhance responding to the transfer CS, but that this transfer is usually incomplete-the occasion setter usually has less effect on the transfer CS than on the CS with which it was trained (e.g., Davidson \& Rescorla, 1986; Holland, 1986a, 1989a, 1989b; Rescorla, 1985; see Swartzentruber, 1995, for a review).

Theories suggesting that occasion setters are CS specific predict that they should not transfer at all; nevertheless such theories can account for these results in other ways. One is simply that, because both feature and target stimuli will have formed direct associations with the US, summation might occur; in other words, responding to the target CS might be enhanced when it is preceded by an excitatory feature stimulus because of a contribution from the associative strength governed by that feature. A second reason for predicting transfer is obtained by considering stimulus generalization. The prediction of no transfer is based on the assumption that the animal discriminates training and transfer CSs perfectly; in reality, however, it is quite likely that the animal will confuse these two stimuli to some extent. If this is so, then the occasion setter will have some effect on responding to the transfer CS, although this will never be as great as its effect on the original, training stimulus. There is, in fact, a reasonable amount of support for this suggestion. In some studies, for example, the training and transfer stimuli were in the same modality, and in others they shared various temporal characteristics (e.g., Davidson \& Rescorla, 1986; Holland, 1986a, 1989b; Rescorla, 1985); both of these factors might have fostered stimulus generalization. Moreover, although some authors have argued that generalization does not occur between the stimuli they have chosen, such arguments usually refer solely to the physical characteristics of the stimuli, and do not take account of the fact that general- 
ization between training and transfer CSs might have been enhanced by their common training history (cf. Lamarre \& Holland, 1987; Wilson \& Pearce, 1990). There is now, however, substantial evidence that a common training history will enhance generalization (e.g., Hall, Ray, \& Bonardi, 1993; Honey, 1990; Honey \& Hall, 1989), and that this occurs during occasion-setting training-it has recently been reported that generalization between two stimuli is enhanced if they have both been the targets of different occasion-setting discriminations (Bonardi \& Hall, 1994). In short, there are at least two reasons why, even if occasion setters were CS specific, some transfer might occur.

An alternative to the view that the occasion setter acts on the entire CS-US association is Rescorla's (e.g., 1985) suggestion that the occasion setter acts directly on the US representation, facilitating the degree to which it can be activated by the CS. This account predicts that the occasion setter will show transfer because it will enhance responding to any CS that is associated with that particular US; indeed, all other things being equal, the occasion setter should be just as effective with the transfer CS as with the original target stimulus. (Holland, 1989a-see also Lamarre \& Holland, 1987-has also proposed an account that makes this prediction, but only provided that the transfer CS comes from another occasion-setting discrimination.) As is clear from the preceding discussion, the data from transfer studies provide partial support for these theories in that some transfer does occur. What these theories need to explain is why that transfer is frequently incomplete. One way they can do this is to suggest that the transfer CS suffers generalization decrement. In a transfer test, the transfer CS is accompanied by the occasion setter for the first time, and it is possible that for this reason the animal perceives the CS as a partially novel stimulus. This generalization decrement effect, either by reducing the responding the transfer CS can elicit or by enhancing the rate of extinction to the transfer CS, could make it look as though transfer had been attenuated. A more refined version of this hypothesis has been proposed by Holland (e.g., 1989b), who has argued that, during occasion-setting training, an association is likely to form between the occasion setter and its target CS. He suggests that, on transfer test trials, this association will allow the occasion setter to evoke a representation of the original target CS while the transfer CS is being presented; this, he argues, could induce generalization decrement in the transfer CS, and so attenuate the transfer that is observed. In support of this proposal he has reported that presentations of the occasion setter alone, which should reduce the strength of its association with the target CS, increase the amount of transfer that is obtained (e.g., Holland, 1989b).

The logic behind the present experiments was that all theories can accommodate the fact that some transfer occurs--either by arguing that occasion setters act on the US and so should show transfer, or by saying that although they act on the CS-US association, some trans- fer could be produced by summation and stimulus generalization. We focused instead on the fact that these two types of theory have different explanations for the finding that transfer is often incomplete-theories asserting that occasion setters act on the CS-US association predict imperfect transfer directly, whereas those claiming that occasion setters act on the US must appeal to generalization decrement to explain imperfect transfer effects. Therefore, to support the idea that occasion setters act on the CS-US association, there is a need for evidence that transfer will be imperfect (if not absent) even when generalization decrement is unlikely to have occurred or cannot provide a complete explanation of the effect. The aim of the present experiments, which used pigeons as subjects, was to provide such evidence. Experiment 1 was designed to obtain an imperfect transfer effect using a procedure which we hoped was unlikely to foster generalization decrement; Experiments 2 and 3 were designed to confirm that the effect observed in Experiment 1 could not be explained in terms of generalization decrement.

Experiment 1 used keylight CSs and diffuse stimuli as occasion setters. Rescorla (1985) has found imperfect transfer with such a procedure, but in his experiment the diffuse occasion setter overlapped with the keylight target. The transfer test trials therefore entailed presentation of a novel simultaneous compound of the target keylight with the transfer feature; if the presence of the transfer feature altered the animals' perception of the keylight, this might well have induced a generalization decrement effect. In this experiment, the likelihood of such generalization decrement was reduced by ensuring that the occasion setter always terminated before the onset of the target keylight; in this way, the transfer test trials would not involve presenting the target keylight in a simultaneous compound that the animal had never experienced before. It should be acknowledged, however, that if this procedure does yield imperfect transfer, the effect might still be attributable to generalization decrement; this could arise by means of an association between the feature and the target, in the manner proposed by Holland (e.g., 1989b) or simply because the aftereffects of the feature stimulus are able to alter perception of the target CS on transfer test trials. Experiments 2 and 3 therefore aimed to provide more compelling evidence that imperfect transfer cannot necessarily be explained in terms of generalization decrement.

\section{EXPERIMENT 1}

A within-subject procedure was employed, the design of which is summarized in Table 1. A single group of pigeons received training in which presentations of two keylight targets, $x$ and $y$, were followed by the delivery of grain on some occasions but not on others. Every reinforced presentation of $x$ and of $y$ was preceded by one of two diffuse feature stimuli, a tone and a flashing light; the tone signaled reinforced presentations of $x$, and the light signaled reinforced presentations of $y$. The features 
Table 1

Design of Experiment 1

\begin{tabular}{cccc}
\hline & \multicolumn{3}{c}{ Test } \\
\cline { 2 - 4 } Training & Same & Different & Target \\
\hline $\begin{array}{c}\text { Tone }(x \rightarrow \text { food }) \\
x \rightarrow \text { nothing } \\
\begin{array}{c}\text { Flash }(y \rightarrow \text { food }) \\
y \rightarrow \text { nothing }\end{array}\end{array}$ & Tone $(x)$ & Flash $(x)$ & $x$ \\
\hline
\end{tabular}

Note-Tone and flash were 10 -sec stimuli immediately preceding presentation of the keylights, $x$ and $y$, as shown. The keylight presentations were all of 5-sec duration, as were the grain presentations on reinforced trials. The $x$ and $y$ were purple, yellow, star, and triangle, and were counterbalanced (see text).

and targets did not overlap, but the offset of the feature coincided with the onset of the target. Unsignaled presentations of the keylights, which were always nonreinforced, also occurred. After the animals had learned this discrimination and were responding more on signaled than on unsignaled trials, they were tested. There were three types of test trial: (1) same trials, on which $x$ and $y$ were preceded by their own signals, the tone and the flashing light, respectively; (2) different trials, on which each target was preceded by the signal from the other discrimination $-x$ by the flashing light and $y$ by the tone-and (3) trials on which $x$ and $y$ were presented alone. It was anticipated that some transfer would occurthat the birds would respond more to the targets on different trials when they were signaled by the "wrong" feature than when they were presented alone; as we have seen, both theoretical accounts described above can accommodate this result. It was also anticipated that this transfer would be incomplete-that the birds would respond more on same trials than on different trials. As suggested above, the details of the procedure used in this experiment make it unlikely, although by no means impossible, that this imperfect transfer effect could be due to generalization decrement.

\section{Method}

\section{Subjects}

The subjects were 16 locally obtained pigeons maintained at $80 \%$ of their free-feeding body weights (mean ad-lib weight of $463 \mathrm{~g}$; range, $331-613 \mathrm{~g}$ ). They were housed in pairs in a colony room that was lit from 0800 to 2200 each day. They had served in a previous experiment in which the autoshaping procedure was used, but were naive to all the stimuli used in the present experiment.

\section{Apparatus}

Training was conducted in four standard three-key pigeon chambers supplied by Campden Instruments Ltd. Only the center key was used. An in-line projector behind the key allowed the following stimuli to be presented: yellow, purple, star, and triangle. The colors were presented over the entire key (a 2.5 -cm-diam circle). The other stimuli consisted of white figures against a dark background, presented in the center of the key. The triangle was $1 \mathrm{~cm}$ equilateral and upright, and the star was produced by superimposing a $1-\mathrm{cm}$ square and a $1-\mathrm{cm}$ diamond. A grain feeder positioned just above floor level and immediately below the center key was used to provide reinforcement. Except when the flashing light stimulus was presented, each chamber was dimly illuminated by a $2.8-\mathrm{W}$ bulb rated for $24 \mathrm{~V}$ but operated at $15 \mathrm{~V}$. In two of the chambers, this houselight was situated in the ceiling; in the other two, it was situated directly above the center keylight, behind a flange. The flashing light was produced by operating the houselight alternately at $24 \mathrm{~V}$ (i.e., bright) for $0.5 \mathrm{sec}$ and then at $0 \mathrm{~V}$ (i.e., dark) for $0.5 \mathrm{sec}$. A speaker mounted on the front wall could be used to deliver an $80-\mathrm{dB} 1-\mathrm{kHz}$ tone, produced by a Campden Instruments Ltd tone generator. Each chamber was enclosed in a sound-attenuating shell; the operation of an extractor fan provided background masking noise. The equipment was controlled by a BBC microcomputer programmed in a version of BASIC.

\section{Procedure}

Preliminary training. The birds' previous experience of autoshaping meant that all would eat readily from the feeder and peck at illuminated response keys, so they received only a single reminder session of autoshaping at the start of the experiment. This consisted of twenty 10 -sec presentations of a white center key, each of which was followed by a 5-sec presentation of grain; the intertrial interval (ITI) was variable, with a mean value of $120 \mathrm{sec}$ (range, $95-140 \mathrm{sec}$ ). This ITI schedule was retained for the remainder of the experiment.

Occasion-setting training. There followed 20 sessions of occasion-setting training, in which all birds were trained on two feature-positive discriminations; all birds received two sessions per day. The features were the two diffuse stimuli, tone and flashing light; the target stimuli, $x$ and $y$, were keylights. Reinforced trials began with a $10-\mathrm{sec}$ presentation of the feature; this was immediately followed by a 5 -sec presentation of the keylight, which was in turn immediately followed by a 5 -sec presentation of grain. The $x$ was always signaled by the tone, and $y$ always by the flashing light. Nonreinforced trials consisted simply of 5-sec keylight presentations. The $x$ was purple for a quarter of the animals, yellow for a quarter, the star for a quarter, and the triangle for the remaining quarter. If $x$ was a color, $y$ was a shape, and vice versa. Thus, for those animals for whom $x$ was purple or yellow, $y$ was star or triangle; for half of the animals for whom $x$ was purple, $y$ was the star; for the other half, it was the triangle. Star and triangle were counterbalanced across yellow in the same way. Conversely, for the animals for whom $x$ was the star or the triangle, $y$ was either purple or yellow. For half of those for whom $x$ was the star, $y$ was purple; for the other half, it was yellow. Purple and yellow were counterbalanced across the triangle in exactly the same way. This left, for each animal, two keylights that were not employed as targets in these discriminations; these stimuli, which will be referred to as $p$ and $q$, were used later in a further test, and were consistently followed by grain presentations throughout these training sessions. As the training and testing of $p$ and $q$ is not relevant to the present experiment, responding to these stimuli will not be discussed below.

There were 24 trials in each session. In the first six sessions, there were 4 of each type of trial; however, acquisition of the discrimination was poor, so in an attempt to rectify this the proportion of negative trials was increased in the remaining sessions. Each of the next 14 sessions therefore comprised 3 tone- $x$-food and 3 flashing light- $y$-food trials, 6 trials each of $x$ and $y$ alone, and 3 reinforced trials with each of $p$ and $q$. The different types of trial were presented in a quasi-random order.

Test. Four test sessions followed, each consisting of 24 trials. In each session, there were four presentations of $x$ and of $y$ presented alone, four of $x$ preceded by the tone and four of $y$ preceded by the flashing light (same trials, because $x$ and $y$ were preceded by their own features), and four of $x$ preceded by the flashing light and four of $y$ preceded by the tone (different trials, because $x$ and $y$ were each preceded by the feature from the other's discrimina- 
tion). No reinforcement was delivered in these sessions, which were in all other respects identical to those of the occasion-setting training.

\section{Results and Discussion}

Inspection of the data revealed that there were no systematic differences in responding to the keylights according to whether they had been signaled by the flashing light or the tone; thus, the response rates reported below are pooled across these different trial types. The birds virtually never responded during the diffuse signals themselves. The counterbalancing of the experiment was such that four different groups of birds were trained and tested with different pairs of stimuli (see above), and this, although not substantially affecting the pattern of responding, appeared to contribute to the variance in the data. Consequently, counterbalanced subgroup was included as a factor in the analyses that follow. Finally, a significance level of $p<.05$ was employed throughout this and the subsequent experiments.

\section{Occasion-Setting Training}

By the end of this stage, the birds had learned both discriminations and were responding more to the keylights when they were preceded by the features than when they were presented alone. In the last session of this stage, the mean rate of responding to the keylights was 61.75 responses per minute (rpm) on reinforced trials, and $33.44 \mathrm{rpm}$ on nonreinforced trials; an analysis of variance (ANOVA) performed on these data with trial type (reinforced or unreinforced) and counterbalancing as factors revealed that this difference was statistically significant $[F(1,12)=13.61]$; nothing else was significant $\left(F_{\mathrm{S}}<1\right)$.

\section{Test}

The test data from the four test sessions are presented in Table 2. It may be seen that, although the absolute level of responding declined over sessions, the pattern of responding during the different types of trial was the same in each test session. Performance on the original discriminations was maintained; responding to the target keylights was higher on same trials when they were preceded by the features with which they had been trained than when they were presented alone. The question of interest was what happened on different trials, when each target was preceded by the feature from the other discrimination. Responding on different trials was substantially higher than responding on target-alone

Table 2

Response Rates (Responses per Minute) on Same Trials, Different Trials, and Target-Alone Trials in Test Sessions of Experiment 1

\begin{tabular}{lcccr}
\hline & \multicolumn{4}{c}{ Session } \\
\cline { 2 - 5 } \multicolumn{1}{c}{ Trials } & 1 & 2 & 3 & \multicolumn{1}{c}{4} \\
\hline Same & 40.50 & 40.41 & 8.07 & 15.84 \\
Different & 35.91 & 26.72 & 5.07 & 10.97 \\
Target alone & 10.59 & 10.50 & 1.79 & 7.41 \\
\hline
\end{tabular}

trials, suggesting that the occasion setters did facilitate responding to the transfer targets. What was of interest here, however, was the fact that this transfer was incomplete; responding was lower on different trials than on same trials. This description of the data is supported by the results of an ANOVA with trial type (same, different, or target alone), sessions, and counterbalancing as factors. This revealed a significant effect of trial type $[F(2,24)=19.42]$ and sessions $[F(3,36)=17.69]$, and a significant interaction between these two factors $[F(6,72)=6.47]$; nothing else was significant [largest $F(6,24)=2.13]$. The significant interaction was explored further with an analysis of simple main effects; this revealed that the effect of trial type was significant in Sessions 1 and $2[F(2,24)=16.94$ and 12.85 , respectively] but not in Sessions 3 and $4[F(2,24)=3.04$ and 2.90 , respectively; both $p s<.1]$. Newman-Keuls tests were performed on the data from Sessions 1 and 2 . These revealed that responding on both same and different trials was greater than responding to the target alone in both of these sessions; responding on same trials was greater than responding on different trials in Session 2.

The results of this experiment suggest that the occasion setter's properties showed substantial transfer to the target from the second discrimination-responding was greater on different trials than on trials with the target alone. But the point of interest in the present context was that this transfer was incomplete-responding was greater on same trials than on different trials in the second test session. Both classes of occasion-setting theory can explain this pattern of results, but do so in different ways. The CS specificity accounts, which predict no transfer at all, explain the responding on different trials in terms of summation or generalization between the training and transfer targets. Conversely, those accounts that do not predict CS specificity, and hence do predict transfer, explain the failure to obtain perfect transfer in terms of generalization decrement of the transfer target. Although it was argued above that generalization decrement is less likely to occur with the procedure used here than in experiments in which feature and target overlap (e.g., Rescorla, 1985), this possibility cannot by any means be ruled out. Experiment 2 was therefore designed to provide a control for generalization decrement, in order to distinguish between these alternative interpretations.

\section{EXPERIMENT 2}

Experiment 2 employed a within-subjects design. A single group of animals received training on two occasionsetting discriminations, exactly as in the previous experiment. The features were a clicker and a flashing light; the clicker signaled that $x$ would be reinforced and the flashing light that $y$ would be reinforced; both $x$ and $y$ were nonreinforced when presented alone. After the birds had learned these two discriminations, they were given a transfer test exactly as in the previous experiment. It was anticipated that the birds would again show imperfect transfer and respond more on same trials than on differ- 
ent trials. The question of interest was whether this was, at least in part, because the CS specificity of the occasion setter made it less effective on different trials or solely because the occasion setter induced generalization decrement in the transfer CS on these trials. An attempt was made to answer this question by examining responding to a second pair of keylights that had not been targets of an occasion-setting discrimination. Birds were trained with a second pair of stimuli, $p$ and $q$, on a pseudooccasion-setting discrimination that employed the same feature stimuli as the original occasion-setting discrimination. Targets $p$ and $q$ were treated like $x$ and $y$ in that the click consistently signaled reinforced presentations of $p$ and the flashing light reinforced presentations of $q$; but, although $p$ and $q$ were, like $x$ and $y$, also presented alone, these unsignaled stimulus presentations were always reinforced. In the final test, responding to $p$ and $q$ was examined in exactly the same way as had responding to $x$ and $y$; in other words, the birds experienced same trials, in which $p$ and $q$ were preceded by their own signals, click and flash, respectively, and also different trials, in which each was preceded by the alternative signal. Targets $p$ and $q$ were also presented alone. The design is summarized in Table 3.

The pseudo-occasion-setting training procedure has frequently been used as a control in experiments on occasion setting (e.g., Davidson \& Rescorla, 1986; Holland, 1986b; Rescorla, 1985; Wilson \& Pearce, 1989, 1990). This is because the feature from such a procedure provides no information about whether its target will be reinforced, and so should not become an occasion setter. Therefore, there cannot be any difference in responding on same and different trials with the pseudo-occasion-set targets due to the feature's occasion-setting power over these stimuli, as it has none. But the feature will still be able to produce generalization decrement in the pseudooccasion-set transfer CSs on different trials. It will there-

Table 3

Design of Experiment 2

\begin{tabular}{|c|c|c|c|}
\hline \multirow[b]{2}{*}{ Training } & \multicolumn{3}{|c|}{ Test } \\
\hline & Same & Different & Target \\
\hline \multicolumn{4}{|l|}{$\overline{\mathrm{OS}}$} \\
\hline $\begin{array}{c}\text { Click }(x \rightarrow \text { food }) \\
x \rightarrow \text { nothing }\end{array}$ & Click $(x)$ & Flash $(x)$ & $x$ \\
\hline $\begin{array}{c}\text { Flash }(y \rightarrow \text { food }) \\
y \rightarrow \text { nothing }\end{array}$ & Flash $(y)$ & Click $(y)$ & $y$ \\
\hline \multicolumn{4}{|l|}{ POS } \\
\hline $\begin{array}{c}\text { Click }(p \rightarrow \text { food }) \\
p \rightarrow \text { food }\end{array}$ & Click $(p)$ & Flash $(p)$ & $p$ \\
\hline $\begin{array}{l}\text { Flash }(q \rightarrow \text { food }) \\
\quad q \rightarrow \text { food }\end{array}$ & Flash $(q)$ & Click $(q)$ & $q$ \\
\hline
\end{tabular}

Note-Click and flash were 10 -sec stimuli immediately preceding presentation of the keylights, $x, y, p$, and $q$, as shown. The keylight presentations were all of 5 -sec duration, as were the grain presentations on reinforced trials. The keylights were red, green, horizontal, and triangle, and were counterbalanced (see text). OS refers to the fact that $x$ and $y$ were trained in an occasion-setting discrimination, and POS to the fact that $p$ and $q$ were trained in a pseudo-occasion-setting discrimination. fore serve as a control, because the amount of generalization decrement on different trials with the pseudooccasion-set targets $p$ and $q$ should be the same as that on different trials with the occasion-set targets, $x$ and $y$. This is because in each of these cases the feature is followed by an unexpected transfer target, and it is not clear why the aftereffects of the feature should affect the perception of the unexpected occasion-set target more than that of the unexpected pseudo-occasion-set target. This same conclusion follows from Holland's (1989b) more specific explanation of the generalization decrement effect; according to that account, during training an association forms between the click, for example, and the two keylights it signals, $x$ and $p$. On different trials, when the click is followed by one of the transfer targets, the click will evoke representations of both $x$ and $p$, which supposedly induce generalization decrement in the transfer target that is presented. But these representations will be evoked regardless of whether the transfer target is $y$, which has been occasion set, or $q$, which has not, and accordingly the resulting generalization decrement should be the same in both cases.

In summary, it was predicted that the animals would respond more to the occasion-set targets $x$ and $y$ on same trials than on different trials. If this is solely the product of generalization decrement on different trials, then exactly the same decrement in responding should be observed on different trials with the pseudo-occasion-set targets $p$ and $q$. But if the incomplete transfer observed with $x$ and $y$ is in part due to the CS specificity of the features' occasion-setting power over these stimuli, then the difference in responding on same and different trials should be substantially greater with the occasion-set targets, $x$ and $y$, than with the pseudo-occasion-set targets, $p$ and $q$.

\section{Method}

\section{Subjects}

The subjects were 16 locally obtained pigeons maintained at $80 \%$ of their free-feeding body weights (mean ad-lib weight of $484 \mathrm{~g}$; range, 375-556 g). They were maintained as in Experiment 1 . They had served in a previous experiment in which the autoshaping procedure was used, but were naive to all the stimuli used in the present experiment.

\section{Apparatus}

The apparatus used was identical to that employed in Experiment 1 , with the following exceptions. The keylight stimuli used were green, red, triangle, and horizontal striations. The triangle was the same as that used in the previous experiment, and the horizontal striations consisted of three parallel white stripes, $2 \mathrm{~mm}$ wide, $2 \mathrm{~mm}$ apart, and $1 \mathrm{~cm}$ long. The colors were presented in the same manner as in the previous experiment. The diffuse stimuli were the flashing light, which was identical to that used in the previous experiment, and an $80-\mathrm{dB} 20-\mathrm{Hz}$ clicker produced by the Campden Instruments Ltd sound generator referred to previously.

\section{Procedure}

Preliminary training. All birds received two reminder sessions of autoshaping, which were identical to those given in the previous experiment. 
Training. There followed 32 sessions of training, in which all birds were trained on two occasion-setting discriminations with $x$ and $y$ and on two pseudo-occasion-setting discriminations with $p$ and $q$. The features for both sets of discriminations were the two diffuse stimuli, click and flashing light; the target stimuli, $x, y, p$, and $q$ were keylights. Presentations of all four keylights were always reinforced when they were signaled by a feature; $x$ and $p$ were always signaled by the click, and $y$ and $q$ by the flashing light. These trials were otherwise identical to the reinforced trials of the previous experiment. Each of these keylights was also presented alone; $x$ and $y$ were always nonreinforced on these trials, as in the previous experiment, whereas $p$ and $q$ were followed by a 5 -sec delivery of grain. For half of the animals, the occasion-set targets $x$ and $y$ were red and green and the pseudo-occasion-set targets $p$ and $q$ were horizontal and triangle; for the other half, this arrangement was reversed. For half of each of these subgroups, red and horizontal were signaled by the click and green and triangle by the flashing light; for the other half, this arrangement was reversed.

There were 24 trials in each session. Throughout training there were two of each of the four types of signaled trials and four of each of the four types of unsignaled keylight presentations; these different types of trial were presented in a quasi-random order. In all other respects, the sessions in this stage were identical to the training sessions in the previous experiment.

Test. A single test session, consisting of 24 trials, followed. Half of these trials were designed to examine transfer of occasion setting. For this purpose, there were: two presentations of $x$ and two of $y$, target-OS trials (OS = occasion setting); two of $x$ preceded by the click and of $y$ preceded by the flashing light, as had occurred during training, same-OS trials; and four transfer trials, two of $x$ preceded by the flashing light and two of $y$ preceded by the click, different-OS trials. The remaining trials, with $p$ and $q$, were designed to examine the corresponding comparisons with the pseudo-occasion-setting discrimination. Thus, there were: two presentations of $p$ and two of $q$, target-POS trials (POS $=$ pseudo occasion setting); two of $p$ preceded by the click and of $q$ preceded by the flashing light, as had occurred during training, same-POS trials; and four transfer trials, two of $p$ preceded by the flashing light and two of $q$ preceded by the click, different-POS trials. No reinforcement was delivered in this session, which was in all other respects identical to the test sessions of the previous experiment.

\section{Results and Discussion}

Inspection of the data revealed that there were no systematic differences in responding to the keylights according to whether they had been signaled by the flashing light or the click; hence, the response rates that are reported below are pooled across these different trial types. As in the previous experiment, the birds virtually never responded during the diffuse signals themselves.

\section{Training}

By the end of this training stage, the birds had learned the two occasion-setting discriminations and were responding more to $x$ and $y$ when they were signaled by the click and the flashing light than when they were presented alone; during the last session of this stage, the mean rate of responding to $x$ and $y$ was $85.3 \mathrm{rpm}$ when they were signaled by the diffuse stimuli and $15.19 \mathrm{rpm}$ when they were presented alone. The animals also responded appropriately to $p$ and $q$ from the pseudo-occasion-setting discriminations, responding at the same rate to these two keylights regardless of whether or not they were signaled by the diffuse stimuli. The mean rate of responding to $p$ and $q$ was $69.9 \mathrm{rpm}$ when signaled by the click and the flashing light and 65.8 when presented alone. An ANOVA with discrimination (occasion-setting or pseudo-occasionsetting) and trial type (whether or not a signal was present) supported the description of the data that has just been outlined, revealing a significant main effect of discrimination $[F(1,15)=6.88]$, a significant main effect of trial type $[F(1,15)=62.77]$, and a significant interaction between these two factors $[F(1,15)=36.59]$. This interaction was explored using simple main effects, and revealed that responding on signaled trials was significantly higher than on unsignaled trials in the occasionsetting discrimination $[F(1,15)=60.93]$, but not in the pseudo-occasion-setting discrimination $(F<1)$.

\section{Test}

The results of the test are presented in Figure 1. The results from the occasion-setting discrimination mirror those found in Experiment 1. First, the animals continued to perform accurately on the discrimination, responding more on same-OS trials than on target-OS trials; second, substantial transfer was again obtained, the animals responding more on different-OS trials than to $x$ and $y$ alone. But the focus of interest was the fact that, as in the first experiment, this transfer was incom-
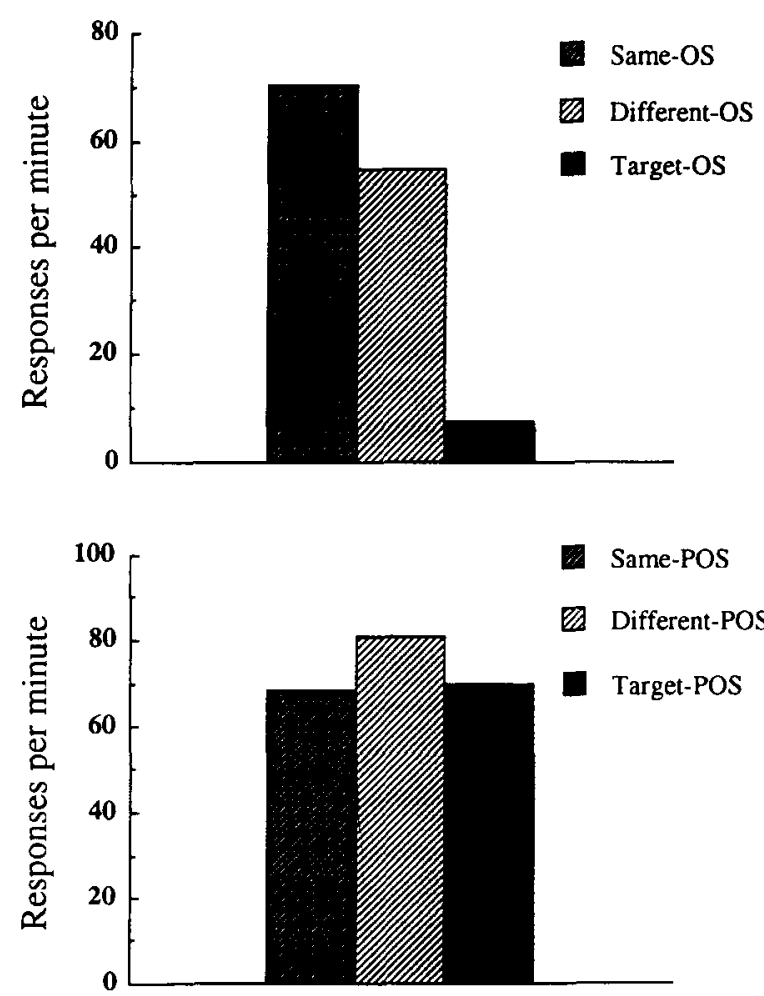

Figure 1. Mean rates of responding during the occasion-set (OS) keylights $x$ and $y$ and during the pseudo-occasion-set (POS) keylights, $p$ and $q$, when preceded by their own features (same trials), when preceded by the feature from the other discrimination (different trials), and when presented alone in the test session of Experiment 2. 
plete; responding on same-OS trials was greater than responding on different-OS trials. A very different pattern of results was obtained in the pseudo-occasion-setting discrimination condition, however. The birds continued to respond at approximately the same rate to $p$ and $q$ on same-POS trials, when they were preceded by their own signals, as when they were presented alone; however, instead of responding more on same-POS trials than on different-POS trials as they did in the occasion-setting condition, they actually responded less.

The critical result was the difference in the pattern of responding on same and different trials with the targets from the two types of discrimination. This was therefore analyzed further with a factorial ANOVA, with discrimination (occasion setting or pseudo occasion setting) and trial type (same or different) as factors. This revealed no effect of discrimination $[F(1,15)=3.44]$ or of trial type $(F<1)$, but did produce a significant interaction between these two factors $[F(1,15)=12.18]$. This interaction was explored using simple main effects; this revealed that responding was significantly higher on same-OS trials than on different-OS trials $[F(1,15)=$ 8.79]; although responding was numerically higher on different-POS trials than on same-POS trials, this fell just short of statistical significance $[F(1,15)=4.36, p=$ .054].

Further analysis confirmed that performance on the original discriminations was maintained. An ANOVA with discrimination (occasion setting or pseudo occasion setting) and trial type (same or target alone) as factors revealed a significant main effect of discrimination $[F(1,15)=16.67]$ and of trial type $[F(1,15)=24.81]$, and also a significant interaction between these two factors $[F(1,15)=45.09]$. This interaction was examined further with simple main effects, and revealed that responding between the two types of trial differed in the occasion-setting discrimination condition $[F(1,15)=$ 39.40] but not in the pseudo-occasion-setting discrimination $(F<1)$.

The results of this experiment provide good evidence that the effects of an occasion setter are CS specific. In accord with this notion, in the test with the occasion-set targets, animals responded more on same trials than on different trials. But, as argued in Experiment 1, to adopt this interpretation, one must be sure that the difference observed was not due solely to generalization decrement of the transfer CS. The test with the pseudo-occasion-set targets provided an answer to this question by giving an estimate of the amount of generalization decrement occurring on the critical trials with the occasion-set targets. If the latter result is due solely to generalization decrement, then exactly the same difference should be observed with the pseudo-occasion-set targets. It was not. If anything, the animals responded more, rather than less, on different trials with the pseudo-occasion-set targets. It seems that the imperfect transfer found with the occasionset cues is not to be explained in terms of generalization decrement.
As noted above, the animals in the pseudo-occasionsetting group responded more on different trials than on same trials, and this effect was close enough to statistical significance to deserve some comment. One possible explanation might lie in the suggestion made by Wagner (e.g., 1981) that the degree to which a stimulus is processed may differ according to whether or not it is surprising. According to this theory, predicted stimuli are processed more poorly than those that are surprising; this poorer processing affects a number of things, one of them being the amount of conditioned responding that the stimulus may elicit-a predicted stimulus will elicit less conditioned responding than a surprising one. Some empirical support for this so-called "negative priming effect" comes from the results of a recently reported set of experiments by Honey, Hall, and Bonardi (1993). Animals were trained with a serial conditioning procedure, in which A signaled reinforced presentations of $X$ and $B$ signaled reinforced presentations of $Y$. In a subsequent test, $\mathrm{X}$ and $\mathrm{Y}$ elicited less conditioned responding when each was preceded by its "own" signal (by A and B, respectively) than when preceded by the signal for the other stimulus (when $\mathrm{X}$ was preceded by $\mathrm{B}$, and $\mathrm{Y}$ by $\mathrm{A}$ ).

Applying this principle to the present results could explain the effect observed in the pseudo-occasion-setting discrimination condition. As argued above, it is probable that in discriminations of this type each target CS comes to be predicted by the feature that precedes it during training. This means that during the test, the target CSs will be less surprising on same trials, when they are predicted by their respective features, than on different trials, when they are not. This leads to the prediction of more responding on different trials than on same trials, exactly the result that was obtained. There is every reason to suppose, of course, that exactly the same negative priming process went on in the test of the occasion-set targets; but this is not inconsistent with the interpretation presented here. For the occasion-set cue, priming would tend to increase responding on different trials, whereas the specificity of the occasion setter to its original target would tend to reduce it. The present results suggest that the latter effect is more powerful than the former, producing the overall result of more responding on same trials than on different trials. If anything, the presence of this opposing priming effect means that the difference in responding due to the target specificity of the occasion setter is being underestimated.

\section{EXPERIMENT 3}

Before we can conclude that occasion setters are CS specific, an alternative interpretation of the results of Experiment 2 must be considered. In that experiment it was argued that the occasion-set and pseudo-occasionset targets would suffer equal amounts of generalization decrement during the test. But, in making that argument, it was implicitly assumed that generalization decrement of a stimulus was independent of that stimulus's training 
history, and this is not necessarily the case. For example, in Experiment 2, the occasion-set targets were reinforced on some occasions and not on others, whereas the pseudooccasion-set targets were always reinforced. This difference in their reinforcement history might have had some influence on how generalization decrement affected responding to these stimuli; perhaps responding to a stimulus that has always been reinforced is less susceptible to the effects of generalization decrement than one that has a history of nonreinforcement. It would be far more compelling, therefore, to replicate these findings under conditions in which occasion-set and pseudo-occasion-set targets have had a similar training history. Experiment 3 was designed to achieve this.

A single group of birds received training that was essentially identical to that received in Experiment 2. Thus, two diffuse features served as occasion setters for two keylight targets, $x$ and $y$, and as pseudo-occasion-setters for two further keylights, $p$ and $q$. In addition, in order to equate as far as possible the treatment of these two pairs of keylights, it was arranged that $x, y, p$, and $q$ would be reinforced and nonreinforced on an equal number of occasions during training. To achieve this, a third diffuse stimulus, a red houselight, was employed; on additional trials, presentation of this red light signaled reinforced trials with $x$ and $y$ and nonreinforced trials with $p$ and $q$. After training on this discrimination, the animals were tested exactly as before. The design is summarized in Table 4.

It was predicted that the target diffuse stimuli would become occasion setters for $x$ and $y$ exactly as in the previous experiment; although these keylights were also reinforced when signaled by the red light, this should not prevent the target diffuse stimuli from signaling that $x$

Table 4

Design of Experiment 3

\begin{tabular}{|c|c|c|c|}
\hline \multirow[b]{2}{*}{ Training } & \multicolumn{3}{|c|}{ Test } \\
\hline & Same & Different & Target \\
\hline \multicolumn{4}{|l|}{ OS } \\
\hline $\begin{array}{c}\text { Noise }(x \rightarrow \text { food }) \\
x \rightarrow \text { nothing } \\
\operatorname{Red}(x \rightarrow \text { food })\end{array}$ & Noise $(x)$ & Dark $(x)$ & $x$ \\
\hline $\begin{array}{l}\text { Dark }(y \rightarrow \text { food }) \\
y \rightarrow \text { nothing } \\
\operatorname{Red}(y \rightarrow \text { food })\end{array}$ & Dark $(y)$ & Noise $(y)$ & $y$ \\
\hline \multicolumn{4}{|l|}{ POS } \\
\hline $\begin{array}{l}\text { Noise }(p \rightarrow \text { food }) \\
p \rightarrow \text { food } \\
\operatorname{Red}(p \rightarrow \text { nothing })\end{array}$ & Noise $(p)$ & Dark $(p)$ & $p$ \\
\hline $\begin{array}{l}\text { Dark }(q \rightarrow \text { food }) \\
\quad q \rightarrow \text { food } \\
\quad \operatorname{Red}(q \rightarrow \text { nothing })\end{array}$ & Dark $(q)$ & Noise $(q)$ & $q$ \\
\hline
\end{tabular}

Note-Noise, dark, and red were 10-sec stimuli immediately preceding presentation of the keylights, $x, y, p$, and $q$, as shown. The keylight presentations were all of $5-\mathrm{sec}$ duration, as were the grain presentations on reinforced trials. The keylights were blue, orange, grid, and circle, and were counterbalanced (see text). OS refers to the fact that $x$ and $y$ were trained in an occasion-setting discrimination with noise and dark, and POS to the fact that $p$ and $q$ were trained in a pseudooccasion-setting discrimination with noise and dark. and $y$ would be reinforced. Likewise it was predicted that the target diffuse stimuli would acquire no control over responding to $p$ and $q$; although $p$ and $q$ were nonreinforced when signaled by the red light, this did not influence the fact that the target diffuse stimuli provided no information as to whether or not these keylights would be reinforced. It was anticipated that, in the test, the animals would respond more to the occasion-set targets $x$ and $y$ on same trials than on different trials, as they had in Experiments 1 and 2. As in Experiment 2, if this loss of responding on different trials were due to generalization decrement, then exactly the same effect should be observed with the pseudo-occasion-set targets $p$ and $q$, as generalization decrement for these stimuli should be equated. But if the loss of responding to $x$ and $y$ on different trials were due to the fact that occasion setters are in part CS specific, the loss should be substantially more profound with $x$ and $y$ than with the pseudo-occasion-set targets $p$ and $q$. As noted above, this logic relies on the assumption that the differential training history of the occasion-set and pseudo-occasion-set targets does not influence the effect of generalization decrement on responding to these stimuli. This assumption is more realistic in this experiment than in Experiment 2, as here $x$, $y, p$, and $q$ were reinforced and nonreinforced on an equal number of occasions during training.

\section{Method}

\section{Subjects}

The subjects were 16 locally obtained pigeons maintained at $80 \%$ of their free-feeding body weights (mean ad-lib weight of $471 \mathrm{~g}$; range, 363-544 g). They were maintained as in Experiment 1 . They had served in a previous experiment in which the autoshaping procedure was used, but were naive to all the stimuli used in the present experiment.

\section{Apparatus}

The apparatus used was identical to that employed in Experiment 1 , with the following exceptions. For this experiment, the houselight was situated in the center of the ceiling in all the chambers. The keylight stimuli used were blue, orange, a circle, and a grid. The grid consisted of the superimposition of horizontal and vertical striations, each consisting of three parallel white stripes, $2 \mathrm{~mm}$ wide, $2 \mathrm{~mm}$ apart, and $1 \mathrm{~cm}$ long, and the circle was a white disk $1 \mathrm{~cm}$ in diameter. The colors were presented in the same manner as in the previous experiment. There were two visual diffuse stimuli: dark, which was produced by turning off the dim houselight, and red, which was produced by turning on a second 2.8-W bulb situated directly above the center key behind a flange; this bulb, which was operated at $24 \mathrm{~V}$, was covered with a red transparent plastic cover so that it illuminated the box with red light. A Campden Instruments Ltd noise generator was used to produce a third diffuse stimulus, a $79-\mathrm{dB}$ white noise that was pulsed at $1 \mathrm{~Hz}$.

\section{Procedure}

Training. There were 50 sessions of training, in which all birds were trained on two occasion-setting discriminations with $x$ and $y$ and on two pseudo-occasion-setting discriminations with $p$ and $q$. The features for both sets of discriminations were noise and dark; the target stimuli, $x, y, p$, and $q$ were keylights. For half of the animals, the occasion-set targets $x$ and $y$ were blue and orange and the pseudo-occasion-set targets $p$ and $q$ were circle and grid; for 
the other half, this arrangement was reversed. The experiment was counterbalanced exactly as in Experiment 2 . The procedure of the present experiment differed in that, in addition to all the types of trial delivered in the sessions of Experiment 2, both the occasionset targets $x$ and $y$ and the pseudo-occasion-set targets $p$ and $q$ were also presented signaled by the red light; the occasion-set targets were reinforced on these trials, whereas the pseudo-occasionset targets were not. Each red light presentation was $10 \mathrm{sec}$ long, and its offset coincided with the onset of the keylight, the duration of which was $5 \mathrm{sec}$; the duration of grain delivery, when it occurred, was also $5 \mathrm{sec}$ on these trials. Occasion-set and pseudo-occasionset targets were reinforced and nonreinforced an equal number of times in each session; thus $x$ and $y$ were each signaled by the red light twice per session and $p$ and $q$ four times per session. There were 36 trials in each session, with a mean ITI of $90 \mathrm{sec}$ (range, $70-110 \mathrm{sec}$ ). In all other respects, sessions in this stage were identical to the training sessions in the previous experiment.

Test. Two test sessions followed, each of which consisted of 24 trials with a mean ITI of $90 \mathrm{sec}$, exactly as during training. No reinforcement was delivered in these sessions, which were in all other respects identical to the test session of the previous experiment; there were no trials with the red light during the test.

\section{Results and Discussion}

Inspection of the data revealed that there were no systematic differences in responding to the keylights according to whether they had been signaled by the dark or by the noise; thus, the response rates that are reported below were pooled across these different trial types. As in the previous experiment, the birds virtually never responded during the diffuse signals themselves.

\section{Training}

By the end of this training stage, the birds had learned the two occasion-setting discriminations, and were responding more to $x$ and $y$ when they were signaled by the noise and the dark than when they were presented alone; during the last session of this stage, the mean rate of responding to $x$ and $y$ was $88.82 \mathrm{rpm}$ when they were signaled by the diffuse stimuli and $42.28 \mathrm{rpm}$ when they were presented alone. Thus, the occasion-setting discrimination was clear, although not as large as in the previous experiment. The animals responded at approximately the same rate to $p$ and $q$ when they were signaled by the noise and dark as when they were presented alone, the corresponding rates being 93.00 and $98.72 \mathrm{rpm}$, respectively. An ANOVA with discrimination (occasionsetting or pseudo-occasion-setting) and trial type (whether the keylights were signaled by the noise and dark or were presented alone) supported the description of the data that has just been outlined, revealing a significant main effect of discrimination $[F(1,15)=5.94]$, a significant main effect of trial type $[F(1,15)=8.16]$, and a significant interaction between these two factors $[F(1,15)=$ 36.46]. This interaction, explored using simple main effects, revealed that responding was significantly higher on signaled trials than on unsignaled trials in the occasionsetting discrimination $[F(1,15)=21.54]$ but not in the pseudo-occasion-setting discrimination $(F<1)$. The animals also responded at a fairly high rate on reinforced trials with $x$ and $y$ when they were signaled by the red light but at a low rate on the nonreinforced trials with $p$ and $q$ when they were signaled by this diffuse stimulus, the corresponding rates being 96.94 and $13.31 \mathrm{rpm}$, respectively.

\section{Test}

The results of the test, pooled over both test sessions, are presented in Figure 2. The pattern of results bore a close resemblance to that of Experiment 2. First, the animals continued to perform accurately on the discrimination, responding more on same-OS trials than on targetOS trials; second, substantial transfer was again obtained, the animals responding more on different-OS trials than to $x$ and $y$ alone. Finally, this transfer was again incomplete; responding was greater on same-OS trials than on different-OS trials. In the pseudo-occasion-setting condition, the birds responded slightly more to $p$ and $q$ on same$P O S$ trials when they were preceded by their own signals than when they were presented alone; as in Experiment 2, they also responded slightly more on different-POS trials than on same-POS trials. The mean response rates for each session are presented separately in Table 5, and it seems clear that the pattern of results was more or less maintained across both test sessions, the only difference being that in Session 1 the birds responded slightly more on
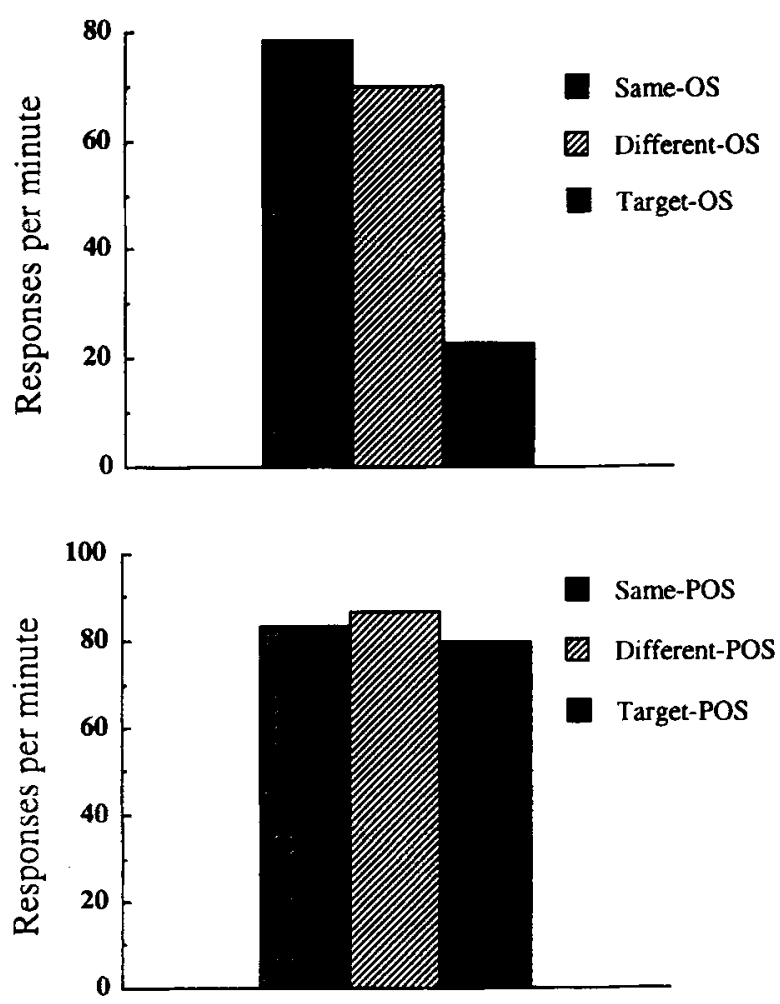

Figure 2. Mean rates of responding during the occasion-set (OS) keylights $x$ and $y$, and during the pseudo-occasion-set (POS) keylights, $p$ and $q$, when preceded by their own features (same trials), when preceded by the feature from the other discrimination (different trials), and when presented alone in the test sessions of Experiment 3. 
Table 5

Response Rates (Responses per Minute) in the Six Types of Test Trial in Each of the Two Test Sessions of Experiment 3

\begin{tabular}{|c|c|c|c|c|c|c|}
\hline \multirow[b]{3}{*}{ Session } & \multicolumn{6}{|c|}{ Trial Type } \\
\hline & \multicolumn{3}{|c|}{ OS } & \multicolumn{3}{|c|}{ POS } \\
\hline & Same & Different & $\begin{array}{l}\text { Target } \\
\text { Alone }\end{array}$ & Same & Different & $\begin{array}{l}\text { Target } \\
\text { Alone } \\
\end{array}$ \\
\hline 1 & 89.25 & 81.56 & 34.31 & 99.35 & 96.56 & 84.75 \\
\hline 2 & 67.69 & 58.50 & 11.25 & 67.50 & 76.50 & 74.25 \\
\hline
\end{tabular}

same-POS trials than on both different-POS and targetalone trials, whereas in Session 2 the reverse was true.

As in Experiment 2, the critical result - the difference in the pattern of responding on same and different trials with the targets from the two types of discriminationwas analyzed with a factorial ANOVA, with discrimination (occasion setting or pseudo occasion setting), trial type (same or different), and sessions as factors. This revealed no effect of discrimination $[F(1,15)=2.42]$ or of trial type $(F<1)$, but did reveal a significant interaction between these two factors $[F(1,15)=14.11]$. There was also a significant main effect of sessions $[F(1,15)=$ 9.49], but this did not interact with any other factor, and nothing else was significant [largest $F(1,15)=2.02$ ] Simple main effects revealed that responding was higher on same-OS trials than on different-OS trials, and that this difference fell just short of statistical significance $[F(1,15)=4.47, p=.052]$. Although, as in Experiment 2 , responding was slightly higher on different-POS trials than on same-POS trials, this was not significant in the present experiment $[F(1,15)=1.25]$.

Performance on the original discriminations was maintained. An ANOVA with discrimination (occasion setting or pseudo occasion setting), trial type (same or target alone), and sessions as factors revealed a significant main effect of discrimination $[F(1,15)=21.68]$ and trial type $[F(1,15)=32.05]$ and a significant interaction between these two factors $[F(1,15)=34.79]$; there was also a significant main effect of sessions $[F(1,15)=19.76]$. Nothing else was significant [largest $F(1,15)=1.94$ ] The significant interaction was examined further with simple main effects, which revealed that responding between the two types of trial differed in the occasionsetting discrimination condition $[F(1,15)=40.93]$ but not in the pseudo-occasion-setting discrimination $(F<1)$.

The results of Experiment 3 were also very similar to those of Experiment 2; in both experiments, the birds appeared to respond more on same trials than on different trials to the occasion-set targets $x$ and $y$, but if anything did the opposite with the pseudo-occasion-set targets $p$ and $q$. This impression was confirmed by the results of a further ANOVA that compared the results of Experiment 2 with the pooled results of Experiment 3, with experiment (Experiment 2 or Experiment 3), discrimination (occasion setting or pseudo occasion setting), and trial type (same or different) as factors. This revealed a main effect of discrimination $[F(1,30)=5.79]$ and a sig- nificant interaction between this factor and trial type $[F(1,30)=21.15]$; nothing else was significant [largest $F(1,30)=3.70]$. The significant interaction was analyzed further with simple main effects, which revealed that there was a significant effect of trial type for both the occasion-setting and pseudo-occasion-setting discriminations $\left[F_{\mathrm{s}}(1,30)=13.25\right.$ and 5.59 , respectively $]$. The implication is that equating the training history of the occasion-set and pseudo-occasion-set targets had little impact on test performance.

As made clear above, the claim that Experiments 2 and 3 really control for the effects of generalization decrement rests on the assumption that these effects are the same for both occasion-set and pseudo-occasion-set cues. It was argued that in Experiment 2, in which occasionset and pseudo-occasion-set cues had very different reinforcement histories, this assumption was inappropriate. This criticism is less relevant to the present experiment, in which the numbers of reinforced and nonreinforced trials with the two types of stimulus were equated and yet a virtually identical pattern of results was obtained. But, although the most obvious difference between the occasion-set and pseudo-occasion-set cues of Experiment 2 was eliminated in the present experiment, one could still argue that other differences between the two types of stimulus remained. For example, the occasionset targets $x$ and $y$ were trained in a feature-positive arrangement, whereas the pseudo-occasion-set targets, being reinforced when presented alone but nonreinforced when signaled by the red light, were effectively trained in a feature-negative arrangement. It could be that stimuli trained in feature-positive and feature-negative tasks are differentially sensitive to generalization decrement. If one were to assume that the cue from a feature-positive discrimination was more susceptible to generalization decrement than both a continuously reinforced cue and also that from a feature-negative discrimination, one could explain the entire pattern of results. Although there are no independent reasons for making this assumption, neither is there good reason to reject it. We must therefore conclude that, although these results rule out some interpretations of a generalization-decrement account of imperfect transfer effects, they cannot eliminate all of them. But, to the extent that these experiments make a generalization decrement account less plausible, they add to the evidence in favor of the conclusion that occasion setters are specific to their target CSs. 


\section{GENERAL DISCUSSION}

The results of these experiments provide evidence in support of the suggestion that, at least with the training procedures used here, imperfect transfer of occasion setting is not to be explained in terms of generalization decrement. This is consistent with theories predicting that occasion setters are CS specific in their action (e.g., Bonardi, 1989; Bouton, 1990). Previous experiments examining transfer of occasion setting have not provided a clear answer to this question, since those instances in which imperfect transfer was obtained were ambiguous; because it is difficult to rule out the possibility of generalization decrement in any given transfer procedure, it is usually more parsimonious to attribute imperfect transfer to generalization decrement than to CS specificity of occasion setting.

The problem with using transfer as a tool to examine whether occasion setters are CS specific is, as we have seen, that evaluating transfer involves comparing responding to the target CS when it is presented with the original or with a second transfer occasion setter. The critical comparison is therefore confounded with whether or not the target CS has been presented with the occasion setter before, and this produces the differential generalization decrement that supposedly accounts for imperfect transfer effects. Although there are ways of finding out whether occasion setters are CS specific that do not involve making such a confounded comparison (e.g., Rescorla, 1991a, 1991b), these can raise other difficulties. For example, Rescorla (1991a) reports an experiment in which two keylights, $x$ and $y$, and four features, A, B, C, and D, were trained in four occasion-setting discriminations, the reinforcement of each keylight being signaled by two different features (i.e., $\mathrm{A} x+x-$, $\mathrm{B} x+x-, \mathrm{C} y+y-, \mathrm{D} y+y-)$. In a subsequent test, feature compounds (e.g., AB, AC, CD, BD) were presented with a third, transfer, keylight which had not previously been experienced with any of the features. The feature compounds were more effective in the same condition, when both had been trained with the same target keylight (i.e., $\mathrm{AB}$ or $\mathrm{CD}$ ), than in the different condition when they had been trained with different keylights (i.e., AC or $\mathrm{BD})$. Rescorla interpreted this as evidence that the animals had encoded which feature belonged to which target, and hence that the features' occasion-setting properties were specific to their original targets. However, these results may be explained by considering the effects of feature-target associations. During training, an association could form between each feature and the target whose reinforcement it signaled (e.g., between $\mathrm{A}$ and $x$, $\mathrm{B}$ and $x, \mathrm{C}$ and $y$, and $\mathrm{D}$ and $y$; cf. Holland 1989b). The existence of these associations would ensure that, during training, each feature would have been accompanied by an expectation, or image, of its own target keylight. In the different condition of the test, $\mathrm{A}$ and $\mathrm{C}$, for example, were presented in compound, each having been trained with different keylights. This would ensure that $A$ would evoke an image of $x$ and $\mathrm{C}$ an image of $y$ during these different trials. Thus, A would for the first time be experienced in compound with an image of $y$, and $C$ for the first time with an image of $x$, and this might have the effect of inducing generalization decrement of the features on these trials. This would not be true of the same condition, in which both features in the compound (e.g., A and B) would evoke an image of the same target keylight, $x$, with which both had been experienced during training. This differential generalization decrement on different trials could conceivably account for the pattern of results that was observed.

Feature-target associations might also be able to account for the results of a second experiment reported by Rescorla (1991b). Animals were trained with two targets, $x$ and $y$, and three features, $\mathrm{A}, \mathrm{B}$, and $\mathrm{C} ; \mathrm{C}$ signaled reinforcement of both targets, whereas $A$ and $B$ signaled reinforcement of only one of the targets (i.e., $\mathrm{A} x+x-$, $\mathrm{C} x+x-, \mathrm{B} y+y-, \mathrm{C} y+y-) . \mathrm{C}$ was then extinguished in compound with one of the target keylights, $x$, and then $A$ and $B$ were tested with a third, transfer keylight which, again, had not previously been experienced with any of the features. It was found that $\mathrm{A}$, whose target keylight $x$ had been extinguished in compound with $C$, was less effective at eliciting responding at test than was $B$, whose target keylight $y$ had not been extinguished in this way. This implies that some aspect of the target was encoded with the feature, implying that the feature was in part specific to its target CS. However, this result can also be accounted for by appealing to the effect of feature-target associations formed during occasion-setting training. By means of such associations, both $\mathrm{A}$ and $\mathrm{B}$ might evoke a representation of their original target keylights, $x$ and $y$, respectively, during presentations of the transfer target in the test. But B's target, $y$, would still have been excitatory, whereas A's target, $x$, would have been extinguished. The evocation of B's excitatory target might therefore produce a summation effect and enhance responding to the transfer keylight, whereas no such summation effect could be produced by the extinguished target evoked by A. This account would predict more responding on trials with B than on those with A-exactly the result that was observed.

In conclusion, it seems that it is extremely difficult to rule out alternative accounts of experiments suggesting that occasion setters are CS specific. The results of the two experiments reported by Rescorla (1991a, 1991b), which attempted to avoid the problems of transfer experiments, can be explained by appealing to the effects of feature-target associations. The present experiments controlled for the effects of feature-target associations, but instead fell prey to the argument that targets treated in different ways might be differentially susceptible to generalization-decrement effects. It seems that the question is by no means settled; nevertheless, one may argue that, in combination, these various experiments provide converging evidence that occasion setters are specific to their target CSs. 
It has been argued that the modulatory properties of occasion setters are the same as those exercised by contexts (e.g., Bouton, 1994; see also Bouton \& Swartzentruber, 1986). If this assumption is correct, then we may also consider the evidence suggesting that conditioning is context specific in appetitive conditioning, one-trial conditioned emotional response conditioning, and flavoraversion procedures (e.g., Bonardi, Honey, \& Hall, 1990; Hall \& Honey, 1989, 1990). These results are also, of course, susceptible to an account in terms of generalization decrement, if one allows that a CS trained in one context might be perceived differently when presented in a second context. Hall and Honey $(1989,1990)$ provided some evidence that this was not a satisfactory explanation of their results. They argued that if the generalization decrement account was correct and the CS was perceived differently in a second context, then the animal was effectively experiencing a novel stimulus, in which case one might expect to see a return of unconditioned responding. However, when they tested this prediction directly, they found no evidence that unconditioned responding returned with a change of context. The present results thus add to a growing body of evidence that occasion setters act, at least in part, on the CS representation (for a review, see Swartzentruber, 1995), and are therefore consistent with the notion that their site of action is the CS-US association itself.

We have so far assumed that the same mechanism is responsible for the solution of all occasion-setting discriminations, and yet this is not necessarily the case. For example, the discriminations involved in the present paper were complex in that, in the second and third experiments, each feature acted both as an occasion setter and as a pseudo occasion setter. It is possible that discriminations like this are solved in a different way from those involving a single feature-target combination. This means that, even if one accepts the evidence from the present studies as suggesting that occasion setters are specific to their targets, this may be true only of the complex discriminations that were used here. It is not obvious how one could rule out such a possibility. On the other hand, until there is independent evidence that different types of discrimination are solved in different ways, one could argue on grounds of parsimony that what is true of the discriminations used in the present experiments is also true of simpler occasion-setting discriminations.

Although we have argued that generalization decrement did not play a substantial role in the present training procedures, it would be wrong to assume that this is generally true. It was mentioned above that Holland (e.g., 1989b) has proposed his own account of imperfect transfer according to which generalization decrement is produced by means of an association between feature and target. Support for this interpretation is provided by his finding that extinction of the feature, and thus of the association between it and the target stimulus, enhances the transfer that is observed (Holland, 1989b). But even if Holland is correct in arguing that his transfer results are adequately explained in terms of generalization decrement, this is not inconsistent with the interpretation presented here. There is no reason to suppose that the two mechanisms accounting for imperfect transfer, generalization decrement and CS specificity of occasion setting, could not operate in the same experiment, and presumably the balance between these two factors will vary with the precise experimental procedures employed. The procedures used in Holland's (1989b) experiment-rat subjects and auditory targets - were very different from those described here. If this, for some reason, made generalization decrement substantial (relative to the effect of the CS specificity of the occasion setters), then this could explain why manipulation of generalization decrement had such a substantial effect on his results. Moreover, it is worth pointing out that, even though he succeeded in enhancing transfer with feature extinction, that transfer did not always become perfect as a result of this manipulation. This may be regarded as evidence that a component of the incomplete transfer was due to the CS specificity of the occasion setter and, hence, was independent of feature extinction. The main import of the present findings, therefore, is that, under appropriate circumstances, evidence for CS specificity of occasion setting may be observed that is not obviously attributable to generalization decrement.

\section{REFERENCES}

BoNaRd, C. (1989). Inhibitory discriminative control is specific to both the response and the reinforcer. Quarterly Journal of Experimental Psychology, 41 B, 225-242.

Bonardi, C., \& HALL, G. (1994). Occasion-setting training renders stimuli more similar: Acquired equivalence between the targets of feature-positive discriminations. Quarterly Journal of Experimental Psychology, 47B, 63-81.

Bonardi, C., Honey, R. C., \& Hall, G. (1990). Context specificity of conditioning in flavor-aversion learning: Extinction and blocking tests. Animal Learning \& Behavior, 18, 229-237.

Bouton, M. E. (1990). Context and retrieval in extinction and in other examples of interference in simple associative learning. In L. W. Dachowski \& C. F. Flaherty (Eds.), Current topics in animal learning: Brain, emotion and cognition (pp. 25-53). Hillsdale, NJ: Erlbaum.

BouTON, M. E. (1994). Conditioning, remembering, forgetting. Journal of Experimental Psychology: Animal Behavior Processes, 20, 219-231.

Bouton, M. E., \& Swartzentruber, D. (1986). Analysis of the associative and occasion-setting properties of contexts participating in a Pavlovian discrimination. Journal of Experimental Psychology: Animal Behavior Processes, 12, 333-350.

DAVIDSON, T. L., \& ResCoRLA, R. A. (1986). Transfer of facilitation in the rat. Animal Learning \& Behavior, 14, 380-386.

HALL, G., \& HoNEY, R. C. (1989). Contextual effects in conditioning, latent inhibition, and habituation: Associative and retrieval functions of contextual cues. Journal of Experimental Psychology: Animal Behavior Processes, 15, 232-241.

Hall, G., \& Honey, R. C. (1990). Context-specific conditioning in the conditioned-emotional-response procedure. Journal of Experimental Psychology: Animal Behavior Processes, 16, 271-278.

HALl, G., RAY, E., \& BoNARDI, C. (1993). Acquired equivalence between cues trained with a common antecedent. Journal of Experimental Psychology: Animal Behavior Processes, 19, 391-399.

Holland, P. C. (1983). Occasion-setting in Pavlovian feature positive discriminations. In M. L. Commons, R. J. Herrnstein, \& A. R. Wagner (Eds.), Quantitative analyses of behavior: Discrimination processes (Vol. 4, pp. 183-206). New York: Ballinger. 
Holland, P. C. (1986a). Temporal determinants of occasion setting in feature-positive discrimination. Animal Learning \& Behavior, 14, 111-120.

Holl.and, P. C. (1986b). Transfer after serial feature positive discrimination training. Learning \& Motivation, 17, 243-268.

Holland, P. C. (1989a). Acquisition and transfer of conditional discrimination performance. Journal of Experimental Psychology: An imal Behavior Processes, 15, 154-165.

Holland, P. C. (1989b). Feature extinction enhances transfer of occasion setting. Animal Learning \& Behavior, 17, 269-279.

HONEY, R. C. (1990). Stimulus generalization as a function of stimulus novelty and familiarity in rats. Journal of Experimental Psychology: Animal Behavior Processes, 16, 178-184.

Honey, R. C., \& HALl, G. (1989). Acquired equivalence and distinctiveness of cues. Journal of Experimental Psychology: Animal Behavior Processes, 15, 338-346.

HoNEY, R. C., HALL, G., \& BoNaRDI, C. (1993). Negative priming in associative learning: Evidence from a serial-conditioning procedure. Journal of Experimental Psychology: Animal Behavior Processes, 19, 90-97.

LAMARRE, J., \& Holland, P. C. (1987). Transfer of inhibition after serial feature negative discrimination training. Learning \& Motivation, 18, 319-342.

Rescorla, R. A. (1985). Conditioned inhibition and facilitation. In R. R. Miller \& N. E. Spear (Eds.), Information processing in animals: Conditioned inhibition (pp. 299-326). Hillsdale, NJ: Erlbaum.

ResCORLA, R. A. (1991a). Combinations of modulators trained with the same and different target stimuli. Animal Learning \& Behavior, 19, 355-360.

Rescorla, R. A. (1991b). Transfer of inhibition and facilitation mediated by the original target stimulus. Animal Learning \& Behavior, 19, 65-70.

Ross, R. T., \& Holland, P. C. (1981). Conditioning of simultaneous and serial feature-positive discriminations. Animal Learning \& Behavior, 9, 293-303.

Ross, R. T., \& Holland, P. C. (1982). Serial positive patterning: Implications for "occasion-setting." Bulletin of the Psychonomic Society, 19, 159-162.

SwartZentruber, D. E. (1995). Modulatory mechanisms in Pavlovian conditioning. Animal Learning \& Behavior, 23, 123-143.

WAGNER, A. R. (1981). SOP: A model of automatic memory processing in animal behavior. In N. E. Spear \& R. R. Miller (Eds.), Information processing in animals: Memory mechanisms (pp. 5-47). Hillsdale, NJ: Erlbaum.

Wilson, P. N., \& Pearce, J. M. (1989). A role for stimulus generalisation in conditional discrimination learning. Quarterly Journal of Experimental Psychology, 41B, 243-273.

WILson, P. N., \& PEARCE, J. M. (1990). Selective transfer of responding in conditional discriminations. Quarterly Journal of Experimental Psychology, 42B, 41-58.

(Manuscript received June 19, 1995;

revision accepted for publication September $6,1995$. 